\title{
Impact of Growth Hormone on Cystatin C
}

\author{
Lisa Sze ${ }^{a}$ d $\quad$ René L. Bernays ${ }^{b, c} \quad$ Cornelia Zwimpfer ${ }^{a}$ Peter Wieslie \\ Michael Brändle ${ }^{d} \quad$ Christoph Schmid ${ }^{a}$ \\ ${ }^{a}$ Division of Endocrinology and Diabetes, and ${ }^{b}$ Department of Neurosurgery, \\ University Hospital, and ' Department of Neurosurgery, Clinic Hirslanden, Zurich, \\ ${ }^{\mathrm{d}}$ Division of Endocrinology and Diabetes, Kantonsspital, St. Gallen, and e Department of \\ Internal Medicine, Kantonsspital Frauenfeld, Frauenfeld, Switzerland
}

\section{Key Words}

Acromegaly $\cdot$ Creatinine $\cdot$ Cystatin C $\cdot$ Growth hormone $\cdot$ Kidney

\begin{abstract}
Background: Cystatin C (CysC) is an alternative marker to creatinine for estimation of the glomerular filtration rate (GFR). Hormones such as thyroid hormones and glucocorticoids are known to have an impact on CysC. In this study, we examined the effect of growth hormone $(\mathrm{GH})$ on CysC in patients with acromegaly undergoing transsphenoidal surgery. Methods: Creatinine, CysC, GH and insulin-like growth factor-1 (IGF-1) were determined in 24 patients with acromegaly before and following transsphenoidal surgery. Estimated GFR was calculated using the Chronic Kidney Disease Epidemiology Collaboration formula. Results: In all patients, surgical debulking resulted in decreased clinical disease activity and declining GH/IGF-1 levels. Postoperatively, biochemical cure was documented in 20 out of 24 patients. Creatinine levels (mean \pm SEM) increased from $72 \pm 3$ to $80 \pm 3 \mu \mathrm{mol} / \mathrm{l}(p=0.0004)$ and concurrently, estimated GFR decreased from $99 \pm 3$ to $91 \pm 3 \mathrm{ml} / \mathrm{min}(p=0.0008)$. In contrast to creatinine, CysC levels decreased from $0.72 \pm 0.02$ to $0.68 \pm 0.02 \mathrm{mg} / \mathrm{l}(p=0.0008)$. Conclusions: Our study provides strong evidence for discordant effects of $\mathrm{GH}$ on creatinine and $\mathrm{Cys} C$ in patients with acromegaly undergoing transsphenoidal surgery, thus identifying another hormone that influences CysC independent of renal function.

(C) 2013 S. Karger AG, Basel
\end{abstract}

\section{Introduction}

Cystatin C (CysC) is an alternative marker to creatinine for estimation of the glomerular filtration rate (GFR), and formulas combining both CysC and creatinine are increasing in popularity [1]. CysC is a sensitive indicator of GFR, as it is freely filtered by glomeruli and almost completely reabsorbed and catabolized by tubular epithelial cells [2]. As compared to 
creatinine, CysC is less dependent on age and gender $[3,4]$. It is considered superior for detection of mild renal dysfunction [5], and for prediction of cardiovascular risk and overall mortality beyond that provided by the GFR [6]. The latter may not just be due to differences in renal handling of creatinine and $\mathrm{CysC}$, but also due to differences in tissue origin and production rates of the two compounds. Indeed, CysC has been found to increase with obesity $[3,7,8]$, to rise in proportion to the number of metabolic syndrome components, and to be associated with insulin resistance $[9,10]$ independent of the GFR.

However, non-GFR determinants of CysC remain poorly defined, as they mainly derive from cross-sectional studies, and associations are notoriously subject to confounding $[3,11]$. Only few intervention studies have addressed potentially non-GFR-dependent CysC-influencing variables, and these often have failed to show an impact of factors such as changes in dietary protein intake and inflammation on CysC $[12,13]$.

It has been shown that CysC may be influenced by hormones independent of renal function. Triiodothyronine stimulates the production of CysC in vitro [14, 15], and thyroid hormones increase its levels in vivo [15-17]. Moreover, CysC production is stimulated by dexamethasone in vitro $[14,18]$ and by pharmacological doses of glucocorticoids in vivo [19]. The effect of growth hormone (GH) on CysC is as yet unknown, but is of great interest because excessive GH production as found in acromegaly not only causes pathognomonic clinical signs, but also leads to lower creatinine levels and an increased GFR [20]. Furthermore, acromegaly is associated with insulin resistance, hyperglycemia and reduction in life expectancy mainly due to cardiovascular disease. Acromegaly is usually caused by a pituitary adenoma, and the main goal of treatment is correction of GH excess, often achieved by transsphenoidal removal of the tumor. The aim of this interventional study was to examine the effect of GH excess and its correction by surgery on CysC and creatinine levels in patients with newly diagnosed acromegaly.

\section{Methods}

\section{Patients}

Twenty-nine consecutive patients with newly diagnosed acromegaly, referred to the University Hospital Zurich between 2006 and 2009, were considered eligible for this study, which was approved by the local ethics committee and conducted in accordance with the Helsinki declaration. Twenty-four subjects (10 female/14 male, aged 28-76 years) gave written informed consent and were included in this prospective case series. Diagnosis of acromegaly was based on typical clinical findings, elevated insulin-like growth factor-1 (IGF-1) concentrations, and non-suppressible GH during a 75-gram oral glucose tolerance test. In all patients, GH excess was caused by a pituitary adenoma. Adenoma size (in $\mathrm{mm}^{3}$ ) estimation and details of the surgical procedure have been reported elsewhere [21]. The initial tumor size ranged from 50 to $5,091 \mathrm{~mm}^{3}$ (median 1,844). Patients underwent intraoperative MRI-guided transsphenoidal surgery as primary treatment for acromegaly, performed by the same surgeon. One patient had undergone previous surgery 17 years ago for prolactinoma with incomplete tumor resection. Clinical examination and laboratory investigations were performed before surgery and at a postoperative follow-up visit 22-124 days (median 88) after surgery. None of the patients suffered from overt central hypothyroidism or hypocortisolism requiring replacement therapy, neither before nor after surgery. Thyroid-stimulating hormone and adrenocorticotropic hormone deficiency were excluded by normal free thyroxine and morning cortisol levels, respectively. Still, at the time of evaluation, 1 patient had received levothyroxine before surgery, and another patient was prescribed hydrocortisone after the operation for safety reasons. 
Sze et al.: Impact of Growth Hormone on Cystatin C

\section{Biochemical Analyses}

Blood samples were drawn after overnight fasting. IGF-1 was determined by an in-house radioimmunoassay, as described in Goede et al. [16], and GH by an immunoradiometric assay (hGH-RIACT; CIS Bio International, Oris Industries, Gif-Sur-Yvette, France). Fasting plasma glucose was measured on a Roche-Hitachi Modular Clinical Chemistry analyzer using commercial tests from Roche Diagnostics. Insulin was determined by a solid-phase radioimmunoassay (CIS Bio International, Oris Industries). Homeostasis model assessment (HOMA) was used to estimate insulin resistance. CysC was measured using an immunologic turbidimetric assay (Cobas Integra 800 system; Roche Diagnostics, Rotkreuz, Switzerland) with DAKO reagents (DAKO Diagnostics, Zug, Switzerland; normal range $0.53-0.95 \mathrm{mg} / \mathrm{l}$, coefficient of variation $5.9 \%$ at $0.97 \mathrm{mg} / \mathrm{l}$ ). Creatinine was measured with the kinetic Jaffe reaction (Hitachi P-Modular system; Roche Diagnostics). Estimated GFR was calculated using the Chronic Kidney Disease Epidemiology Collaboration (CKDEPI) formula, which seems more accurate than the widely used Modification of Diet in Renal Disease Study equation, especially at an estimated GFR $>60 \mathrm{ml} / \mathrm{min} / 1.73 \mathrm{~m}^{2}$ [22]. The CKD-EPI formula, expressed as a single equation, is GFR $=141 \times \min (\mathrm{Scr} / \kappa, 1)^{\alpha} \times$ $\max (\mathrm{Scr} / \kappa, 1)^{-1.209} \times 0.993^{\text {Age }} \times 1.018$ [if female] $\times 1.159$ [if black], where Scr is serum creatinine (mg/dl), $\kappa$ is 0.7 for females and 0.9 for males, $\alpha$ is -0.329 for females and -0.411 for males, min indicates the minimum of Scr/ $/$ or 1 , and max indicates the maximum of Scr/к or 1 .

\section{Statistical Analysis}

Data are presented as means \pm SEM and were normally distributed using the KolmogorovSmirnov test. Accordingly, values before and after surgery were examined using Student's paired $t$ test, and values between genders were compared using an unpaired t test. Correlation coefficients were assessed using Pearson's correlation formula. Statistical analyses were conducted using STATA SE10 software (StataCorp, College Station, Tex., USA). p values $<0.05$ were considered statistically significant.

\section{Results}

Body Mass Index, Blood Pressure, Heart Rate

After surgery, body mass index (BMI), systolic and diastolic blood pressure, heart rate, and pulse pressure decreased considerably (table 1).

\section{Growth Hormone and Insulin-Like Growth Factor-1}

In all 24 patients, surgical debulking resulted in decreased clinical disease activity and declining GH basal and IGF-1 levels. GH nadir levels of $<1 \mu \mathrm{g} / \mathrm{l}$ were documented either in a random blood sample or during an oral glucose tolerance test in 20 of the 24 patients, consistent with complete adenoma removal.

Creatinine, Estimated GFR, and CysC

Creatinine levels increased (from $72 \pm 3$ to $80 \pm 3 \mu \mathrm{mol} / \mathrm{l}, \mathrm{p}=0.0004$; fig. 1), and the estimated GFR declined accordingly (from $99 \pm 3$ to $91 \pm 3 \mathrm{ml} / \mathrm{min}, \mathrm{p}=0.0008$ ), whereas CysC levels decreased (from $0.72 \pm 0.02$ to $0.68 \pm 0.02 \mathrm{mg} / \mathrm{l}, \mathrm{p}=0.0008$ ). The (inverse) correlation between CysC and estimated GFR reached statistical significance only after ( $r=-0.44, \mathrm{p}=$ $0.03)$, but not before surgery $(r=-0.19, p=0.37)$. CysC was higher in men than in women before surgery $(0.75 \pm 0.03$ vs. $0.66 \pm 0.02 \mathrm{mg} / \mathrm{l}, \mathrm{p}=0.03)$, but similar in both groups after surgery $(0.70 \pm 0.03$ vs. $0.65 \pm 0.02 \mathrm{mg} / \mathrm{l}, \mathrm{p}=0.19)$. Creatinine levels were higher in men than 


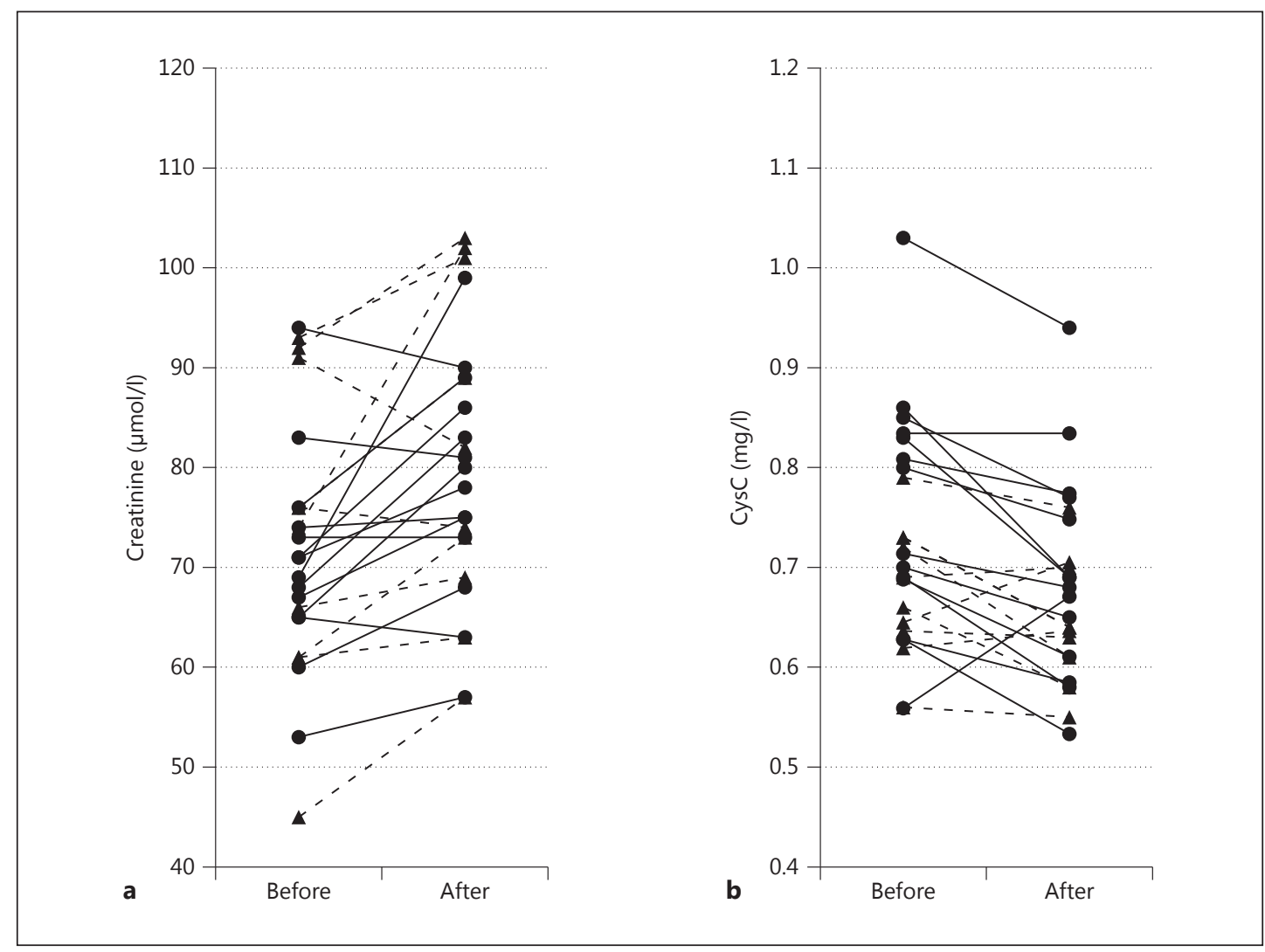

Fig. 1. Creatinine (a) $(p=0.0004)$ and CysC $(\mathbf{b})(p=0.0008)$ before and after surgery. Males = Circles, solid lines; females $=$ triangles, dashed lines.

Table 1. Clinical and biochemical parameters before and after surgery (mean \pm SEM)

\begin{tabular}{lccc}
\hline & Before surgery & After surgery & $\begin{array}{l}\text { p values } \\
\text { (before vs. after) }\end{array}$ \\
\hline BMI & & & 0.02 \\
Systolic blood pressure, mm Hg & $28.1 \pm 0.1$ & $27.6 \pm 0.1$ & $<0.0001$ \\
Diastolic blood pressure, mm Hg & $82.8 \pm 1.8$ & $78.5 \pm 2.4$ & 0.03 \\
Pulse pressure, mm Hg & $56 \pm 3$ & $47 \pm 2$ & 0.0007 \\
Heart rate, bpm & $76 \pm 2$ & $66 \pm 2$ & 0.0007 \\
GH, $\mu$ g/l & $26.3 \pm 5.2$ & $2.5 \pm 0.6$ & 0.0001 \\
IGF-1, $\mu$ g/l & $594 \pm 34$ & $192 \pm 12$ & 0.0001 \\
Phosphate, mmol/l & $1.36 \pm 0.04$ & $1.05 \pm 0.02$ & $<0.0001$ \\
Sodium, mmol/l & $140.4 \pm 0.5$ & $140.9 \pm 0.5$ & $\mathrm{NS}$ \\
Potassium, mmol/l & $3.9 \pm 0.1$ & $4.0 \pm 0.1$ & $\mathrm{NS}$ \\
Fasting plasma glucose, mmol/l & $5.7 \pm 0.2$ & $5.1 \pm 0.2$ & 0.002 \\
HOMA index & $7.4 \pm 0.9$ & $3.3 \pm 0.4$ & 0.0001 \\
CysC, mg/l & $0.72 \pm 0.02$ & $0.68 \pm 0.02$ & 0.0008 \\
Creatinine, $\mu$ mol/l & $72 \pm 3$ & $80 \pm 3$ & 0.0004 \\
GFR, ml/min & $99 \pm 3$ & $91 \pm 3$ & 0.0008 \\
\hline
\end{tabular}


in women both before ( $77 \pm 3$ vs. $63 \pm 3 \mu \mathrm{mol} / \mathrm{l}, \mathrm{p}=0.03$ ) and after surgery ( $88 \pm 3$ vs. $68 \pm 2$ $\mu \mathrm{mol} / \mathrm{l}, \mathrm{p}<0.0001$ ). CysC did not correlate with age, as opposed to creatinine ( $\mathrm{p}=0.01$ before and 0.04 after surgery).

\section{Electrolytes and Glucose Homeostasis}

Fasting plasma glucose and phosphate were high at baseline and decreased significantly following surgery, whereas sodium and potassium remained unchanged. Insulin sensitivity improved after surgery (table 1). Two patients with type 2 diabetes on insulin therapy before surgery were excluded from glucose homeostasis assessment; postoperatively, one of them had normal glucose levels without medication, and insulin was replaced by oral antidiabetic treatment in the other patient. CysC correlated neither with fasting plasma glucose, HOMA index nor BMI.

\section{Discussion}

In our patients with acromegaly, surgical correction of $\mathrm{GH}$ excess resulted in the expected increase in creatinine levels, but decreased CysC levels, pointing out discordant effects of GH excess on creatinine and CysC. Given the known GFR increase caused by GH excess, CysC could have been expected to be low at baseline and to rise after surgery.

Opposite effects on plasma creatinine and CysC have been shown with thyroid hormones [15-17]. Discordant results of these plasma markers have also been found in patients receiving high-dose glucocorticoids as immunosuppressive treatment by some [19], but not all investigators [23]. GH appears to be a further hormone which affects CysC independent of renal function.

Our observation that $\mathrm{GH}$ excess increases CysC is in accordance with the findings during the physiological condition of normal puberty, where GH activity is enhanced. In the NHANES study, CysC levels were found to peak at the age of 12 in females, and at the age of 14 in males, apparently in the absence of changes in the GFR. Since CysC levels rose in parallel with peak height velocity, the authors proposed that CysC levels could be affected by growth in adolescents, and that the age-related variation in CysC levels was likely related to variation in CysC production [24].

We cannot exclude indirect effects of GH to be responsible for the observed changes of CysC levels, e.g. via changes in body composition, insulin resistance, or the generation and action of IGF-1 and triiodothyronine. It could be speculated that acromegaly-associated insulin resistance would be consistent with higher CysC levels before surgery. An association of CysC with insulin resistance has been found in several studies $[9,10]$; however, in our patients, CysC did not correlate with the HOMA index.

Non-GFR-dependent effects of GH on CysC are possibly more readily observed in patients with otherwise unimpaired renal function. The (negative) correlation between CysC and estimated GFR (significant only after surgery) is noteworthy, given that the 'true' GFR of most of our acromegalic patients presumably still remained in a 'creatinine-blind' range and could not be reliably estimated by plasma-derived values, neither by the CKD-EPI formula [22] nor by CysC.

A major advantage of our study is GH excess correction solely by surgery, with most patients being cured. Therefore, results of creatinine and CysC were not confounded by drugs such as somatostatin receptor analogues, which may have renal effects. Measurement of creatinine and CysC in the same blood samples ensured the same GH status as well as other potential determinants of these parameters. 
Our study has several limitations. Firstly, GFR was only estimated from (creatininebased) calculations, and not directly measured by inulin clearance or by isotopes, so the 'true' GFR was not determined. However, we tried to estimate GFR with the CKD-EPI formula, which operates best at a higher GFR ( $>60 \mathrm{ml} / \mathrm{min})[22,25]$. Secondly, we did not analyze body composition (decreased BMI during early postsurgical follow-up is most likely dominated by the loss of extracellular water and muscle mass). Finally, we only measured free thyroxine levels in our patients (to exclude hypothyroidism before or after surgery), but free triiodothyronine was not measured, which is increased by GH and may have an impact on both CysC levels and renal function.

To our knowledge, this is the first study providing strong evidence for a differential effect of $\mathrm{GH}$ on creatinine and $\mathrm{CysC}$, two plasma or serum markers of renal function. Thus, a further hormone is being identified, which influences CysC independent of renal function.

\section{Disclosure Statement}

The authors have no conflict of interest to declare.

\section{References}

1 Inker LA, Schmid CH, Tighiouart $\mathrm{H}$, et al: Estimating glomerular filtration rate from serum creatinine and cystatin C. N Engl J Med 2012;367:20-29.

-2 Randers E, Kristensen JH, Erlandsen EJ, Danielsen H: Serum cystatin C as a marker of the renal function. Scand J Clin Lab Invest 1998;58:585-592.

-3 Stevens LA, Schmid CH, Greene T, et al: Factors other than glomerular filtration rate affect serum cystatin C levels. Kidney Int 2009;75:652-660.

4 Weinert LS, Prates AB, do Amaral FB, Vaccaro MZ, Camargo JL, Silveiro SP: Gender does not influence cystatin C concentrations in healthy volunteers. Clin Chem Lab Med 2010;48:405-408.

5 Dharnidharka VR, Kwon C, Stevens G: Serum cystatin C is superior to serum creatinine as a marker of kidney function: a meta-analysis. Am J Kidney Dis 2002;40:221-226.

-6 Toft I, Solbu M, Kronborg J, et al: Cystatin C as risk factor for cardiovascular events and all-cause mortality in the general population. The Tromso Study. Nephrol Dial Transplant 2012;27:2780-2787.

7 Young JA, Hwang SJ, Sarnak MJ, et al: Association of visceral and subcutaneous adiposity with kidney function. Clin J Am Soc Nephrol 2008;3:1786-1791.

8 de Boer IH, Katz R, Chonchol MB, et al: Insulin resistance, cystatin C, and mortality among older adults. Diabetes Care 2012;35:1355-1360.

9 Lee SH, Park SA, Ko SH, et al: Insulin resistance and inflammation may have an additional role in the link between cystatin $\mathrm{C}$ and cardiovascular disease in type 2 diabetes mellitus patients. Metabolism 2010;59: 241-246.

10 Servais A, Giral P, Bernard M, Bruckert E, Deray G, Isnard BC: Is serum cystatin-C a reliable marker for metabolic syndrome? Am J Med 2008;121:426-432.

11 Knight EL, Verhave JC, Spiegelman D, et al: Factors influencing serum cystatin C levels other than renal function and the impact on renal function measurement. Kidney Int 2004;65:1416-1421.

12 Grubb A, Bjork J, Nyman U, et al: Cystatin C, a marker for successful aging and glomerular filtration rate, is not influenced by inflammation. Scand J Clin Lab Invest 2011;71:145-149.

13 Tangri N, Stevens LA, Schmid CH, et al: Changes in dietary protein intake has no effect on serum cystatin C levels independent of the glomerular filtration rate. Kidney Int 2011;79:471-477.

14 Schmid C, Ghirlanda-Keller C, Zwimpfer C, Zoidis E: Triiodothyronine stimulates cystatin C production in bone cells. Biochem Biophys Res Commun 2012;419:425-430.

$\checkmark 15$ Kotajima N, Yanagawa Y, Aoki T, et al: Influence of thyroid hormones and transforming growth factor-beta1 on cystatin C concentrations. J Int Med Res 2010;38:1365-1373.

16 Goede DL, Wiesli P, Brandle M, et al: Effects of thyroxine replacement on serum creatinine and cystatin C in patients with primary and central hypothyroidism. Swiss Med Wkly 2009;139:339-344.

17 Fricker M, Wiesli P, Brandle M, Schwegler B, Schmid C: Impact of thyroid dysfunction on serum cystatin C. Kidney Int 2003;63:1944-1947.

18 Bjarnadottir M, Grubb A, Olafsson I: Promoter-mediated, dexamethasone-induced increase in cystatin C production by HeLa cells. Scand J Clin Lab Invest 1995;55:617-623. 
Sze et al.: Impact of Growth Hormone on Cystatin C

19 Risch L, Herklotz R, Blumberg A, Huber AR: Effects of glucocorticoid immunosuppression on serum cystatin C concentrations in renal transplant patients. Clin Chem 2001;47:2055-2059.

20 Corvilain J, Abramow M, Bergans A: Some effects of human growth hormone on renal hemodynamics and on tubular phosphate transport in man. J Clin Invest 1962;41:1230-1235.

21 Bellut D, Hlavica M, Schmid C, Bernays RL: Intraoperative magnetic resonance imaging-assisted transsphenoidal pituitary surgery in patients with acromegaly. Neurosurg Focus 2010;29:E9.

22 Levey AS, Stevens LA, Schmid CH, et al: A new equation to estimate glomerular filtration rate. Ann Intern Med 2009;150:604-612.

23 Bokenkamp A, van Wijk JA, Lentze MJ, Stoffel-Wagner B: Effect of corticosteroid therapy on serum cystatin C and beta2-microglobulin concentrations. Clin Chem 2002;48:1123-1126.

-24 Groesbeck D, Kottgen A, Parekh R, et al: Age, gender, and race effects on cystatin C levels in US adolescents. Clin J Am Soc Nephrol 2008;3:1777-1785.

25 Earley A, Miskulin D, Lamb EJ, Levey AS, Uhlig K: Estimating equations for glomerular filtration rate in the era of creatinine standardization: a systematic review. Ann Intern Med 2012;156:785-795. 